\title{
EPISTEMOLOGÍA DEL LENGUAJE: ALGUNOS PARADIGMAS EXPLICAN EL FENÓMENO
}

\author{
EPISTEMOLOGY OF LANGUAGE: SOME PARADIGMAS EXPLAIN THIS PHENOMENON
}

\author{
Edna Maria de Oliveira Ferreira \\ Mestre em Educação Agrícola. Professora EBTT - IF Baiano. Doutoranda em Educação - UNR. \\ Professora IFBaiano e UNR. \\ E-mail:edmaof@hotmail.com \\ http://orcid.org/0000-0002-2606-557X \\ César Costa Vitorino \\ Doutor em Letras. Professor da Universidade do Estado da Bahia (UNEB), campus I, Salvador. \\ E-mail: vitorinoleitura66@gmail.com \\ https://orcid.org/0000-0003-4121-004X.
}

\section{Sady Carolina Gayoso Samudio}

Professora de Espanhol no Sistema CCAA, Salvador-BA

E-mail: sadygayoso@gmail.com

https://orcid.org/0000-0001-5214-0097

\begin{abstract}
RESUMEN
Es indiscutible el valor y la importancia del lenguaje como elemento de socialización entre los humanos. El lenguaje fomenta la cultura, o sea, es la base de la cultura en la sociedad. Este artículo objetiva sintetizar el trayecto hecho para que el lenguaje fuera considerado una ciencia, desde las teorías dualistas hasta las teorías lingüísticas contemporáneas, evidenciando posicionamientos filosóficos en cada perspectiva y sus contribuciones para que los estudios del lenguaje lograsen el status actual. Una pesquisa bibliográfica permitió una apreciación de los tres modelos más significativos para el entendimiento del lenguaje y del sentido: el realismo; el mentalismo y el pragmatismo. Se constató que el lenguaje despierta la atención desde los sofistas y de los filósofos griegos, aunque haya divergencias entre ellos, pues el lugar de la esencia para Aristóteles es el alma y para Platón, la esencia está en lo real. Pero para ambos, las palabras, reales o mentales, son esencias. Por lo tanto, se puede entender que las contribuciones para la comprensión del lenguaje y sentido advenidas del pensamiento más antiguo surgen justamente de la tensión entre el esencialismo y el relativismo; y estas discusiones todavía están presentes en la contemporaneidad, a partir de Wittgenstein, Russel, Frege, Habermas, Foucault, Derrida y resultan en los estudios actuales sobre el lenguaje, como la teoría de la enunciación, de los actos de habla, del análisis del discurso, y otras áreas que hacen interface con el lenguaje. Martins (2005), Santaella (2016), Fiorin (2004, 2017) y Demolin y Storto (2017), entre otros teóricos, respaldaron la argumentación en este texto.
\end{abstract}

PALABRAS CLAVE: Epistemología. Lenguaje. Sentido. Conocimiento.

\section{ABSTRACT}

The value and importance of language as an element of socialization among humans is indisputable. Language fosters culture, then it is the basis of culture in society. This article aims to synthesize the journey made for language to be considered a science, from dualistic theories to contemporary linguistic theories, evidencing philosophical positions in each perspective and their contributions for language studies to achieve its current status. A bibliographic research allowed an appreciation of the three most significant models for understanding language and meaning: realism; Mentalism and Pragmatism. It was found that language arouses the attention of the Greek sophists and philosophers, although there are differences between them, since the locus of the essence for Aristotle is the soul and for Plato, the essence is in the reality. But for both, words, real or mental, are essences. Therefore, it can be understood that the contributions to the understanding of language and meaning arising from the oldest thought arise precisely from the tension between essentialism and relativism; and these discussions are still present in 
contemporaneity, starting with Witttgeinstein, Russel, Frege, Habermas, Foucault, Derrida and result in current studies of language, such as the theory of enunciation, of speech acts, of the analysis of speech. speech, and other areas that interface with language. Martins (2005), Santaella (2016), Fiorin (2004, 2017) and Demolin and Storto (2017), among other theorists, supported the argument in this text.

KEY WORDS: Epistemology. Language. Meaning. Knowledge.

\section{RESUMO}

O valor e a importância da linguagem como elemento de socialização entre os humanos são indiscutíveis. A língua fomenta a cultura, ou seja, é a base da cultura na sociedade. Este artigo tem como objetivo sintetizar o percurso feito para que a linguagem seja considerada uma ciência, das teorias dualistas às teorias lingüísticas contemporâneas, evidenciando posições filosóficas em cada perspectiva e suas contribuições para que os estudos da linguagem atinjam seu status atual. Uma pesquisa bibliográfica permitiu uma apreciação dos três modelos mais significativos para a compreensão da linguagem e de significado: realismo; mentalismo e pragmatismo. Verificou-se que a linguagem desperta a atenção dos sofistas e filósofos gregos, embora existam diferenças entre eles, uma vez que o lugar da essência para Aristóteles é a alma e para Platão, a essência está no real. Mas para ambos, as palavras, reais ou mentais, são essências. Portanto, pode-se compreender que as contribuições para a compreensão da linguagem e do sentido advindas do pensamento mais antigo surgem justamente da tensão entre os essencialismo e relativismo; e essas discussões ainda estão presentes na contemporaneidade, a partir de Wittgenstein, Russel, Frege, Habermas, Foucault, Derrida e resultam em estudos atuais sobre a linguagem, como a teoria da enunciação, dos atos de fala, da análise da fala. outras áreas que fazem interface com a linguagem. Martins (2005), Santaella (2016), Fiorin (2004, 2017) e Demolin e Storto (2017), entre outros teóricos, corroboram o argumento neste texto.

Palavras-chave: Epistemologia. Língua. Senso. Conhecimento.

\section{INTRODUCCIÓN}

La lengua (el lenguaje) es lo que mueve al sujeto en el mundo social. La comunicación a partir de las palabras o símbolos arbitrarios, cambió significativamente el contexto donde vivían los humanos. Hablar una lengua es más que solo dominar su léxico, su estructura; es también dominar su cultura para poder construir adecuadamente los enunciados en los momentos de interacción. De ese modo, la lengua es más que solamente estructura y vocabulario, o sea, la lengua es un objeto cultural; es un proceso socio-histórico y cultural; es el producto de una población de individuos que hablan de manera semejante entre ellos.

Cuando el individuo se percibe a sí mismo como capaz de significar o significarse a sí mismo, es decir, como especial y distinto de otros animales, no siempre se da cuenta o intenta buscar comprender cómo, de hecho, esto sucedió/sucede a lo largo del tiempo. Una de las vertientes de los estudios lingüísticos, el análisis del discurso (AD), que entiende el lenguaje como una obra social y simbólica que constituye al hombre y su historicidad (ORLANDI, 2005), vine abordando estos temas y ha contribuido en gran 
medida a demarcar este espacio del lenguaje como mediador entre el hombre y la realidad - contexto natural y social-, relacionando el lenguaje con su exterioridad. Actualmente, con los avances de los estudios sobre lenguaje, se sabe esa y otras “verdades" al respecto del lenguaje y del sentido.

Aquellos que hoy viven bajo las innovaciones tecnológicas constantes, o mismo la teoría de la complexidad que se propone a un análisis más general, más complexo de la realidad social, y direcciona la observación del investigador para la diversidad y los ajustes y adaptaciones entre el hombre y el medio ambiente (MORIN, 2005), probablemente, pueden evaluar la importancia de la lengua en la vida social. Pero serán sorprendidos al intentaren la búsqueda pelas orígenes del lenguaje, pues van a percibir que es largo el camino para salir del pensamiento mítico, llegar al pensamiento racionalista y, a continuación, ir desbravando el conocimiento cada vez más, para explicar cómo se construye el sentido en las enunciaciones.

La lectura de Martins (2005) nos motivó a producir este artículo como producto de nuestras reinterpretaciones, como forma de rescatar, aunque de manera concisa, los conocimientos producidos a lo largo del tiempo y que, en nuestra sencilla opinión, no deberían desenfocarse. Todavía, con el propósito de facilitar la comprensión a los principiantes en los estudios de lengua o, incluso, a los estudiosos de otras áreas que hacen interfaz con los estudios lingüísticos. De esta manera, se pretende sintetizar de forma breve el trayecto ontológico, epistemológico y ético hecho para que el lenguaje sea considerado una ciencia, desde las teorías dualistas hasta las teorías lingüísticas contemporáneas, evidenciando, de manera concisa, posiciones filosóficas en cada perspectiva y sus aportes para que los estudios del lenguaje lograsen el estado actual.

El lenguaje despierta la atención desde los tiempos de los sofistas, de los filósofos griegos. El origen de toda y cualquier ciencia siempre es interesante. En los siglo 427 a 347 a. C., Platón ya consideraba el lenguaje como un medicamento ${ }^{1}$ (dispositivo) capaz de aproximar el hombre al conocimiento. A través de la comunicación y de los diálogos que desarrollamos con otros semejantes se alargan los conocimientos, ya decía Platón. Así, muchas preguntas, a veces extrañas en aquel contexto, fueron hechas para intentar

\footnotetext{
${ }^{1}$ Medicamento sería un término advenido del griego pharmakon que se refiere al veneno, medicina y cosmético. Platón, en su diálogo Fedro, utiliza ese término para aclarar que el lenguaje era comprendido como solución para el conocimiento; veneno, cuando era manipulada o cosmético, cuando utilizada para crear efectos estéticos, sentido figurativo.
} 
llegar a la verdad, ¿hay verdades eternas? ¿ las cosas tienen una esencia fija que nos transcienda? - son algunos ejemplos de preguntas hechas a camino de la búsqueda por la verdad (ALSTON, 1972).

En este punto, además de las cuestiones fundantes de la filosofía, como: ¿ hay una verdad absoluta? ¿ hay vida después de la muerte? ¿ las cosas tienen una esencia fija?, por ejemplo, se propone para reflexionar - en este artículo - las siguientes preguntas que, a continuación, se intentará contestar: ¿ lo que sería epistemología del lenguaje? ¿ de qué manera el lenguaje se convirtió en ciencia? ¿ las ideas iniciales, entre los sofistas y socráticos, aún se hacen presentes de alguna forma entre los modernos y contemporáneos? ¿ cuales son los interrogantes de hoy día, después del establecimiento de las teorías interaccionistas del lenguaje? Así, en este artículo se pondera sobre esos interrogantes.

\section{¿QUÉ ES Y PARA QUÉ SIRVE LA EPISTEMOLOGÍA?}

Un análisis de los términos episteme + logos - que resulta en epistemología, ayuda a comprender que ese término significa "ciencia de la ciencia”, o “filosofía de la ciencia”. En otras palabras, es la teoría del conocimiento, el estudio de los principios, hipótesis o resultados de las varias ciencias existentes, a partir de un punto de vista crítico. Santaella (2016, p. 36) define epistemología "como el estudio de la naturaleza del conocimiento y de la justificación, especialmente, el estudio de los rasgos determinantes, de las condiciones substantivas y de los límites del conocimiento y de la justificación". De ese modo, se arranca del concepto de epistemología para en seguida desarrollar el tema.

Se puede afirmar que esa teoría consiste en la reconstrucción de modo racional del conocimiento científico, del proceso gnoseológico, de la organización, del desarrollo y del rol de los descubrimientos científicos, bajo las orientaciones diversas de la lógica, lingüística, sociología, filosofía, historia. En fin, a partir de una mirada interdisciplinaria, una vez que todo conocimiento científico está sujeto a cambios de órdenes ideológicas, religiosas, políticas u otras (TESSER, 1994).

Se puede comprender por epistemología como el estudio del conocimiento y de la justificación de la creencia. Para Gianella (1995), “la epistemología son las asignaturas que 
desde la filosofía se ocupan del problema de cómo conocemos, del origen, las condiciones y los límites de nuestro conocimiento". Por supuesto, ésta tiene su frontera bien más difusa, como las demás asignaturas. Badaró (2005) propone dos enfoques: un para referir a los estudios del origen y del valor del conocimiento humano de forma amplia y otro más comprometido con la significación del estudio de la ciencia.

El concepto de epistemología, se puede percibir, presenta distintas concepciones. Desde los grandes filósofos grecos hasta el Siglo XVI, Iglesia y cuestiones teológicas siempre estuvieron presentes en las discusiones sobre conocimiento y cultura. En el Siglo $\mathrm{XVII}$, en el periodo lluminista las discusiones sobre epistemologías se inician y se alargan, porque las nuevas ideas empiezan a ser difundidas por los nuevos saberes y nuevas ciencias que eran emergentes. Bacon y Descartes son evidencias cuando se habla en racionalismo. Bacon contribuyó con el método empirista y Descartes presentó su teoría basada en las matemáticas (Racionalismo).

Tesser (1994, p. 33) aclara que podemos considerar la epistemología como el estudio metódico y reflexivo del conocimiento, su organización, su formación, su desarrollo, su funcionamiento y sus productos intelectuales. Así, es "la ciencia de la ciencia" que propone soluciones consistentes, en el campo de la filosofía, para los problemas que van surgiendo en las investigaciones científicas, además de proporcionar reflexiones sobre métodos y teorías, en la búsqueda de separar ciencia auténtica y pseudociencia.

Hay hoy día tres concepciones de epistemología: el neopositivismo, la interpretación metafísica y el racionalismo científico, de los cuales descienden ideas y teorías que conllevan al conocimiento científico, y que son más influyentes o actuantes. Experiencia y lenguaje se complementan en el neopositivismo, valorizando la ciencia como camino posible al conocimiento (BADARÓ, 2005). Ello es influencia del positivismo de Comte, en lo cual los mitos, las creencias y la religión dan lugar al realismo y al objetivismo, como criterios para evaluar el carácter científico.

La interpretación metafísica, otro aspecto epistemológico, encamina la descubierta de forma progresiva a la realidad, comparando hipótesis y leyes. Ya en el racionalismo científico, es el conocimiento científico que organiza el real. O sea, es de la observación que se origina el conocimiento científico. De ese modo, un campo científico 
puede ser evaluado, interpretado o analizado bajo varias concepciones, siempre heterogéneas y cambiantes, jamás fijas.

Sin embargo, tenemos que poner atención a los tres modelos o posturas filosóficas más significativos del punto de vista conceptual para el entendimiento del lenguaje y sentido: a) realismo, que identifica parte de la realidad; b) mentalismo, que representa lo que existe en la mente compartido entre hablantes; c) y pragmatismo, en lo cual la lengua es entendida en el uso que se hace de ella en las prácticas sociales, teniendo en cuenta su carácter histórico-cultural. En este sentido, Fontes (2019, p. 139) plantea que

El lenguaje se entiende como una actividad. El significado es el uso efectivo que hacemos de las expresiones lingüísticas dentro de contextos siempre específicos. Así, entonación, tonalidad, gestos, relaciones preexistentes, etc., pasan a formar parte del conjunto del proceso de significación y deben ser consideradas también cuando reflexionamos sobre el lenguaje.

Y para comprender las teorías de la lingüística contemporánea es necesario que se examine la ascendencia filosófica de esos diferentes paradigmas. En esta perspectiva, Martins (2005, p. 440) argumenta que "Examinar las ascendencias filosóficas de estas distintas visiones generales del lenguaje es, en este sentido, un recurso que puede ser útil para quienes deseen entenderse a sí mismos en el espacio relativamente laberíntico de las teorías lingüísticas contemporáneas". Todos saben que la filosofía siempre se interesó por las cuestiones que se refieren a la "verdad y al sentido".

Las tres visiones o modelos de posturas filosóficas sobre el sentido del lenguaje (realismo, mentalismo y pragmatismo) encuentran apoyo en el sentido común. Pero, los interrogantes hechos sobre la verdad y sobre el sentido llevaron a creer, en cierto momento, que el lenguaje no es una duplicación de la realidad, no hay una correspondencia lógica y obvia entre los nombres y las cosas del mundo real. Por ejemplo: la palabra "el libro" y el objeto que recibe este nombre en el mundo real.

Eso queda más claro cuando, en lugar del libro, se analiza una palabra que expresa una idea abstracta o un pensamiento, a ejemplo de la felicidad. No hay en el mundo real un objeto para representar este nombre/término. Luego, se percibe que no hay una correspondencia biunívoca entre la idea mental de felicidad y la palabra que le da el nombre (MARTINS, 2005). 
A ese respecto, Martins (Id, p. 470) dice "Esta [concepto esencialista del lenguaje] parece haberse convertido, en efecto, en nuestra comprensión del sentido común: la sorpresa que solemos experimentar cuando falla la comunicación nos da una medida de cómo un sistema de representación de significados esenciales fijos y compartidos." El pragmatismo retira el sentido inmanente de las palabras, pues hay el abandono de la idea de ver los significados cómo cosas de un mundo real o mental y pasa a valorizar el uso cultural que se hace de las palabras, entonces, el lenguaje pasa a ser comprendida como praxis determinada por la cultura, historia y contexto comunicativo.

A continuación, otra duda se le surge a la sociedad: la cuestión relacionada a los sentidos que una misma palabra tiene en diferentes contextos de uso. Se llega a conclusión de que el uso de la lengua es más una deliberación comunitaria y no una acción individual que depende solo del deseo de alguien, o sea, es cuestión contractual (GUILLÉN, 2006).

De ese modo, los tres modelos o paradigmas filosóficos griegos lograron éxitos y pudieron corresponder a las necesidades de determinado momento, cada uno a su modo y a su tiempo, inclusive, en la contemporaneidad. No obstante, queda claro que se está delante de una cuestión filosófica de gran consistencia a discusión sobre lenguaje y el sentido. Todavía hoy se pregunta: ¿Hay una verdad absoluta? ¿Hay vida después de la muerte? ¿Las cosas tienen una esencia fija? La búsqueda por las respuestas continua, tanto del punto de vista filosófico como del pragmático.

A partir de ahí, la filosofía del lenguaje ocupa una posición central en la teoría del método filosófico para evidenciar una teoría del uso o de la significación de una palabra o de una forma de enunciado. Asimismo, las investigaciones continuaron a hacer ciencia, siempre utilizando el lenguaje como medio para llegarse a nuevas ciencias o aun investigando el propio lenguaje, como todavía se hace, a ejemplo de las teorías de la enunciación, los actos de habla, la lingüística textual, análisis del discurso o tantas otras áreas de conocimiento que hacen interface con la lingüística.

Actualmente, las principales líneas de investigación en epistemología son la epistemología genética de Piaget, conocida como psicogénesis interdisciplinaria y que explica la constitución de la formalización lógico-matemáticas del desarrollo humano; la epistemología histórica de Bachelard, para quién la ciencia es histórica y ella nace y desarrolla en situaciones históricas, entonces, el conocimiento es temporal; y, por último, 
la epistemología crítica de Habermas y la arqueológica de Foucault, en la cual el hombre trae su historicidad. Foucault fundamenta sus estudios en las ciencias humanas y plantea que el pensamiento, la cultura y el lugar que la ciencia ocupa en el espacio del saber son determinantes para analizar las relaciones de poder en la sociedad.

Resumiendo, se puede comprender que todas esas epistemologías (desde los griegos, pasando por la epistemología racionalista crítica de Popper - Neopositivismo-, cuyo principio básico es la verificación del valor de las teorías científicas, además de Wittgenstein, Russel, Frege y de la epistemología crítica de Habermas - que presenta interrogantes sobre las responsabilidades sociales de los científicos y de los técnicos y plantea que la ciencia tiene dos ejes: el eje del saber y el eje del poder - o todavía pasando por Bachelard, Derrida, Foucault, en fin...) contribuyen para que nos preguntemos: ¿hay una ciencia hoy?¿cuál es el rol de la ciencia hoy?

O sea, aunque los avances tengan ocurrido, todavía no tenemos respuestas fijas par estos mismos interrogantes. La realidad es cambiante y complexa. Hay que tener eso en cuenta: las respuestas de hoy pueden no servir para el porvenir.

\section{DEL PENSAMIENTO MÍTICO PARA EL PENSAMIENTO RACIONAL}

Las explicaciones míticas de la realidad entre los diferentes pueblos de la antigüedad fueron muy valiosas y precedieron el surgimiento de la filosofía. Sin embargo, no se debe aceptar como verdad que el pasaje del mítico para el racional se deba a una sucesión de fases históricas aisladas. El adviento de la filosofía es mejor comprendido, cuando se lo acepta como la incorporación de un nuevo modo de estar en el mundo, de vivir y de pensar frente a la perplejidad (MARTINS, 2005).

Marcondes (2011) caracteriza el pensar mítico como recurrente a un discurso no justificable, por exigir adhesión a las narrativas o por apelar al sobrenatural. Por otro lado, el pensar filosófico se caracteriza como aquél que renuncia al sobrenatural y es señalado por el carácter crítico, por un discurso justificable y, por lo tanto, abierto a discusiones. Esta definición se mantiene fiel a la idea de la cuál surge el concepto de filosofía.

En fin, el pasaje del pensamiento mítico para el pensamiento racional no fue un momento histórico bien demarcado. Lo más importante es reconocer que él señala un período de insatisfacción con las explicaciones y los parámetros para explicar las 
verdades de la realidad. A partir de ahí, coexisten dos polarizaciones: los sofistas y los filósofos dichos socráticos. Entonces, va a imperar la idea de que sofistas son individuos sin ética y manipuladores del lenguaje y de la opinión ajena. El sentido peyorativo del término "sofistas" queda evidente como si fueran personas que pretendían defender solo sus intereses. Pero Martins (op. cit, p. 447-8) observa que

Comprender ese antagonismo supone en primero lugar abandonar el estigma (...) asociado a los sofistas, segundo el cuál eses maestros de la retórica y de la oratoria que corrían la ciudad con sus enseñanzas no eran más que individuos venales, sin ética - inescrupulosos manipuladores del lenguaje y de la opinión (...) para defender sus propios intereses en las (...), los debates, en las leyes y los rumos de los acontecimientos civiles en general.

Este prejuicio aún existe, pero ha sufrido algunos cambios. Hoy ya tenemos quién comprenda a los sofistas como personas de gran contribución intelectual.

Entonces, se nota que la cisión, polarización entre sofistas y socráticos se da con respecto a la cuestión ontológica. O sea, sobre la naturaleza de los propios seres: ¿Las cosas tienen una esencia permanente? Una respuesta negativa será dada por los sofistas ("el hombre es la medida de todas las cosas") y una respuesta positiva puede ser esperada por los socráticos. La aceptación de una o de otra respuesta va a interferir en cuestiones éticas, políticas y epistemológicas. Finalmente, "si la verdad prevalece sobre el consenso o si el consenso prevalece sobre la verdad, es la consistente controversia entre sofistas y socráticos", plantea Harris y Taylor (1989 apud MARTINS, 2005, p.466).

Se debe mucho a la evolución de las ciencias para los filósofos y sofistas. Y es en el campo de la lingüística donde se puede percibir con más clareza esos avanzos y esa búsqueda por comprender la cognición. Lèvi-Strauss (1955, p. 9) advierte que la lingüística ocupa una posición de privilegio, pues

[...] clasificada entre las ciencias humanas, tiene por objeto, sin embargo, un hecho social, pues el lenguaje no sólo implica la vida en sociedad, también la funda; ¿qué sería una sociedad sin lenguaje? Además, es el más perfecto y complejo de esos sistemas de comunicación que constituyen toda la vida social, y que todas las ciencias sociales - cada una en su nivel particular - se proponen estudiar.

Queda evidente la importancia del lenguaje para la construcción y sustentación de la vida en sociedad. Guillén (2006, p. 71-72) también plantea que, al estudiar el fenómeno del lenguaje, es común buscar una descripción, como, por ejemplo 
Verlo como una positividad con la cual quedan cifradas las posibilidades de la representación isomórfica de lo real o del ser con que se enfrenta el hombre en su experiencia común (...) establecer la relación existente entre la vivencia del conocimiento al que se hace el sujeto en su experiencia en el mundo de la vida [como experiencia pre- predicativa] y la significación que se expresa en el orden apofántico ${ }^{2}(. .$.$) caracterizar la$ formación [génesis] de los actos de creación de las significaciones en la experiencia individual y en la colectiva (...) dirigir la comprensión de lo que implica y significa el uso del lenguaje en términos tanto de la acción comunicativa como de las interacciones que tienden directamente hacia la "efectuación de la historia".

En fin, el lenguaje hace parte de las prácticas o acciones comunicativas y de las interacciones entre los individuos. O sea, se acepta fácilmente hoy que "el sujeto se constituye en el lenguaje y a través de ella" (ORLANDI, 2005).

De ese modo, para que haya una buena comprensión de las teorías del lenguaje nacidas en la lingüística, a ejemplo del estructuralismo, funcionalismo, interaccionismo, se necesita entender cuestiones ontológicas que tienen a ver con la idea de la verdad y del sentido y sus impactos en el pensamiento lingüístico. A continuación, se tiene el embrión del pensamiento pragmático del lenguaje y del sentido, que es el pensamiento sofista, basado en el relativismo (MARTINS, 2005). Pero, ¿cómo eso sucedió? Martins (Id, p. 450, trad. nuestra) plantea que

el legado filosófico más recurrente atribuido a los sofistas es aquel del relativismo - ya aludido a la tesis radical de la imposibilidad de establecerse verdades universalmente válidas, autónomas con respeto a las circunstancias concretas, contingentes y variables de la experiencia humana.

Se comprende que el relativismo imposibilita una aprensión de la realidad como de hecho ella es en sí misma, como se presenta. Si no hay acceso al real, entonces se puede afirmar que el lenguaje revela las opiniones e impresiones consensuales y volátiles de los hombres, estableciendo el lenguaje. De ahí, se puede decir que el pensamiento sofístico es el embrión de una visión pragmática del lenguaje y del sentido.

Lo que se percibe es que los sofistas abren espacios para creerse que las expresiones significan no porque representan algo por sí solas, no porque traen un

\footnotetext{
2 El término greco "apofantikós" tiene su uso relacionado a la lógica aristotélica y suele traducirse por un discurso declarativo o enunciativo. En filosofía se refiere al tipo de discursos en el cual se afirma o niega algo.
} 
sentido inmanente, sino porque no se distancian de los temas humanos, de las prácticas, con efectos previsibles, pero jamás garantizados previamente (MARTINS, 2005).

Así, el lenguaje, la verdad, el sentido y el mundo siempre despertaron la atención desde la antigüedad y continúan a atraer de igual modo hoy delante del pensamiento moderno o contemporáneo. Es constante la búsqueda por saber si tal significado es verdadero o falso, si determinado conocimiento (afirmación) es verdadero o falso. Demolin y Storto (2017, p. 215, trad. nuestra) plantean que

La investigación sobre la evolución del lenguaje, como vamos a ver adelante, trata de reunir el máximo de informaciones posible desde los datos de la genética, del desarrollo infantil, de las neurociencias, de la paleontología, de la antropología, de la psicología comparativa, de la tipología lingüística, de la lingüística historia y de la modelaje matemáticas con el propósito de construir una visión coherente de la facultad del lenguaje humana y su origen.

Entonces, tanto en la modernidad como en la contemporaneidad aún se busca explicar sobre la evolución y el origen del lenguaje. Frege (2002), pensador contemporáneo, dividió el contenido de las expresiones en dos componentes: el sentido y el significado. El sentido sería la expresión del pensamiento que ella expresa, o sea, la referencia que hace, o aún los modos de representaciones de los objetos en el mundo. $Y$ el valor de la verdad estaría asociado a esa capacidad de los objetos de hacer esa referencia. Es muy compleja la discusión sobre la verdad y el sentido del lenguaje. Aquí se hace solo una breve revisión que pasa por los sofistas, por la ontología dualista de Platón (ser sensible y ser inteligible) y por la reflexión sobre la naturaleza del lenguaje.

El otro modelo a ser tratado aquí es el mentalista. Platón y Aristóteles - combaten los sofistas y, a veces, parecen mantener afinidades. Con respecto a la concepción de lenguaje, por ejemplo, defienden que hay un vínculo objetivo y estable que une las palabras a los nombres/términos extralingüísticos que los designan. Ahora bien, ¿cómo queda el sentido figurado, metafórico de las palabras? ¿ hay una violación, una transgresión del sentido normal cuando se utiliza del sentido metafórico? ¿ cómo entender esa situación? Es una referencia directa al sentido figurado y no inmanente de las palabras.

Para Platón, la idea es objeto de cognición; para Aristóteles, la idea es el medio de la cognición. O sea, lo real para Platón, en el mundo aparente, es variable; y en el mundo 
esencial, es fijo. Para Aristóteles, que confiere dimensión prioritaria a la Lógica3, el hombre es localizado/situado en el espacio en lo cual hay dispersiones y variabilidades que se reducen a las esencias universales y autónomas (MARTINS, 2005).

En esta perspectiva, más recientemente, tenemos a Derrida que plantea que la naturaleza del lenguaje ofrece la condición de consciente distinción entre lo que es propio y literal o abstracto o intelectual, oponiéndose a figurado, concreto o sensorial. Entonces, para ese autor, esa consciencia va desde la forma como se concibe la naturaleza hasta la forma como se concibe el pensamiento y los discursos (MIGUENS, 2007).

El par metafórico no-metafórico, o metáfora-concepto, es así más una de las divisiones que jerarquizan y que organizan la forma como un tal pensamiento concibe la naturaleza del pensamiento, una división que el intuito "marginal" del pensamiento que Derrida pretende desmembrar. Por otras palabras, la naturaleza del lenguaje ofrece condiciones de distinción entre metáfora y concepto (MIGUENS, Id).

Foucault (1995), con su "arqueología" de los discursos, busca especificar las condiciones que facilitan históricamente hablar sobre determinada manera acerca de determinado asunto (por ejemplo la locura, o la sexualidad). La relación con la teoría del lenguaje es esa misma: "discursos" son para Foucault las cosas dichas, en contextos históricos de repetición de enunciaciones dadas las regularidades definen las formaciones discursivas', y es eso que se trata de analizar (MIGUENS, Id).

Como señala Martins (2005, p. 472), "la ciencia del lenguaje ocupa en ese escenario una posición especialmente delicada, pues toma como objeto el propio nerviosidad contemporáneo de la controversia, el lenguaje". El lenguaje ocupa el centro del debate actualmente. De ahí, tal vez, ser difícil para la lingüística textual (LT) conceptuar texto, y discurso, pues hay una línea tenue entre ellos: para la LT es un evento materializado, empírico, ya para la $A D$ es un evento sociocognitivo. En fin, el lenguaje hace interdisciplinaridad con las demás ciencias, produciendo ciencia en distintas áreas del conocimiento.

Entonces, no se puede negar la importancia de las reflexiones desde los sofistas hasta hoy, para que se comprenda cómo el lenguaje hace sentido. Al final, está presente

${ }^{3}$ La Lógica se ocupa de los principios que rigen la articulación racional del pensamiento, de lo que hace del hombre un animal racional. 
en todas nuestras acciones: sin el lenguaje no existimos; es nuestra esencia. La comunicación en cualquier campo de conocimiento utiliza el lenguaje; y el mundo se muestra muy avanzado con respecto a las formas de comunicación. Arrancamos de los dibujos en la piedra y llegamos a las más modernas tecnologías de información y comunicación (TIC) - y eso es encantador.

Finalmente, se concluye que además del lenguaje escrito tenemos otras tantas formas (o signos) que mesclan distintas posibilidades, los textos multi-semióticos, y están presentes en todos los contextos culturales, exigiendo siempre una interpretación, comprensión o atribución de significado. Resulta que la ciencia del lenguaje es un campo muy fértil de investigación para producción de conocimientos sobre ella misma y sobre las diversas áreas de estudios.

\section{A MODO DE CIERRE}

La lengua es la base de las interacciones sociales. La lengua es cultura, pues ella determina al individuo o a toda comunidad lingüística una manera peculiar de comprender el mundo a su rededor, siendo influenciada por factores socioculturales e históricos. Hoy tenemos todo ese conocimiento acerca de la lengua, de su estructura, de los sentidos que emana, en fin, de la ciencia del lenguaje, la lingüística, muy valioso para que se comprenda cómo ocurrieran los hechos.

El legado griego para la comprensión de lo que es lenguaje y sentido presenta esos tres modelos: realismo, mentalismo y pragmatismo, que pueden ser resumidos a solo dos, esencialismo o relativismo. La filosofía, sofista y socrática, ha instaurado grandes debates alrededor de la verdad y de las cuestiones existenciales. Pero lo que más marca esas discusiones es, tal vez, la tensión entre el esencialismo y el relativismo.

La perspectiva platónico-aristotélica se respalda en su concepción esencialista del lenguaje y del sentido, fundada en la creencia de que las verdades esenciales son perennes y prevalecen sobre los consensos volátiles de las personas. Por otro lado, la perspectiva sofista, relativista que es, comprende el fenómeno lingüístico vinculado a la idea de que la verdad es mutable y múltiple. O sea, los consensos que regulan las prácticas humanas no son fijos, son cambiantes.

Entonces, el lenguaje no puede ser comprendido como un sistema de representación, pero como una praxis resultante de la cultura, de la historia y por el 
contexto en que se dan los contactos verbales. Se percibe la presencia de la idea de sentido común, tal como le comprenden la filosofía griega, en textos escritos por pensadores modernos y contemporáneos, como: Descartes, Locke, Frege, Russel y hasta mismo en el estructuralismo de Saussure.

Finalizando, se evidencia que la epistemología del lenguaje es marcada por esa tensión entre el esencialismo y el relativismo, olvidado durante siglos en que hubo la hegemonía de la metafísica, y que reaparece con filósofos, en el cambio del siglo XIX; y también con Saussure en el siglo XX, y, todavía, con la presencia de los filósofos contemporáneos, tales como: Wittgenstein, Heidegger, Derrida, Foucault, cuando cuestionan los absolutos metafísicos.

Es decir, el lenguaje es objeto de estudios de la lingüística y, al mismo tiempo, bastante fundamental para el desarrollo humano y para la construcción de otros saberes, para la búsqueda de otras verdades, como mediadora de tantas otras ciencias, ya que es elemento de interacción entre las personas.

En pleno siglo XXI, cuando la información se muestra muy importante, cuando todos vivimos cercado por el lenguaje, es valioso que se discutan esas ideas. Este texto no se propuso profundizar el debate. Lo que se buscó fue provocar discusiones al respecto y traer el tema para el debate y, así, se espera que se lo haya logrado con éxito.

\section{REFERENCIAS}

ALSTON, W. P. A filosofia da linguagem. Rio de Janeiro: Zahar, 1972. Disponível em: http://isabellenobrega.tumblr.com/post/60654753601/do-que-se-trata-a-filosofia-dalinguagem

BADARÓ, C. E. Epistemologia e ciência: reflexão e prática na sala-de-aula. Bauru: EDUSC, 2005.

DEMOLIN, D.; STORTO, L. R. A origem e a evolução da linguagem. In: FIORIN, J. L. (Org.). Novos caminhos da linguística. São Paulo: Contexto, 2017, pp 215- 232.

FIORIN, J. L. Linguística e pedagogia da leitura. In: Revista Linguística e Literatura Scripta, 8 (14), 2004, pp. 107-117. Disponível em: http://periodicos.pucminas.br/index.php/scripta/article/view/12547 
FONTES, N. M. Retórica, Linguagem e Epistemologia: Esboço de uma Teoria Argumentativa do Conhecimento. IN Revista Ágora Filosófica. Recife, v. 19.n. 1, 2019, jan/abr, pp. 135-152.

FOUCAULT, M. A Arqueologia do saber. 6ed. Rio de Janeiro: Forense Universitária, 1995.

FREGE, G. Lógica y Filosofía del lenguaje. Traducción de Paulo Alcoforado. São Paulo:

Cultrix, 2002.

GIANELLA, A. E. Introducción a la Epistemología y Metodología de la Ciencia. La Plata, REUN, 1995.

GUILLÉN, G. V. Tratado de epistemología: fenomenología de las ciencias, la tecnología y la investigación social. 2ed. Bogotá-Colombia: Sociedad de San Pablo, 2006.

LÈVI-STRAUSS, C. Las matemáticas del hombre. Trad. José A. Castorina, publicado en Bulletin International de Sciences Socials. UNESCO, París, vol. 4, 1995.

MARCONDES, D. Textos básicos de filosofia: dos pre-socráticos a Wittgenstein. 7ed. Rio de Janeiro: Zahar, 2011.

MARTINS, H. Três caminhos na filosofia da linguagem. IN MUSSALIM, F.; BENTES, A. C. Introdução à linguística: fundamentos epistemológicos. 2ed. São Paulo: Editora Cortez, 2005, pp 439-473.

MIGUENS, S. Filosofia da linguagem: uma introdução. Ed. Faculdade de Letras da universidade do Porto-Portugal, 2007.

MORIN, E. Ciência com consciência. (8ed). Rio de Janeiro: Bertrand Brasil, 2005.

ORLANDI, E. P. Análise do discurso: princípios e procedimentos. 6ed. São Paulo: Pontes, 2005.

SANTAELLA, L. Por uma epistemologia antidualista. In: LOPES, M. I. V.(org). Epistemologia da comunicação: trajetórias autorreflexivas. São Paulo: ECA-USP, 2016, pp 35- 48.

TESSER, G. J. Principais linhas epistemológicas contemporâneas. IN Revista Educar em Revista- UFPR. Curitiba, n. 10, Jan/Dec., 1994. Disponível em: https://www.scielo.br/scielo.php?script=sci_arttext\&pid=S0104-40601994000100012 